
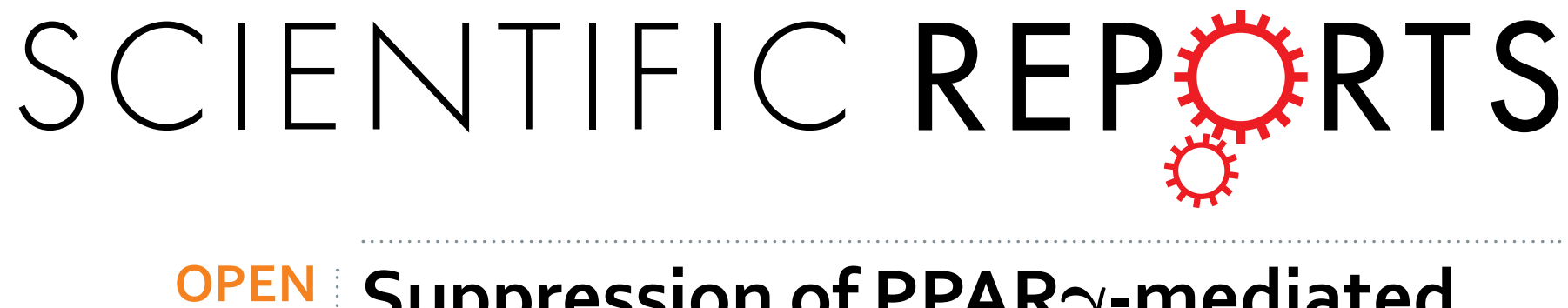

Suppression of PPAR $\gamma$-mediated monoacylglycerol $O$-acyltransferase 1 expression ameliorates alcoholic

Received: 18 March 2016

Accepted: 16 June 2016

Published: 11 July 2016

\section{hepatic steatosis}

Jung Hwan $\mathrm{Yu}^{1,2}$, Su Jin Song ${ }^{1,2}$, Ara Kim ${ }^{1,2}$, Yoonjeong Choi ${ }^{1,2}$, Jo Woon Seok ${ }^{1,2}$, Hyo Jung Kim ${ }^{1}$, Yoo Jeong Lee ${ }^{3}$, Kwan Sik Lee ${ }^{4}$ \& Jae-woo Kim ${ }^{1,2,5}$

Alcohol consumption is one of the major causes of hepatic steatosis, fibrosis, cirrhosis, and superimposed hepatocellular carcinoma. Ethanol metabolism alters the $\mathrm{NAD}^{+} / \mathrm{NADH}$ ratio, thereby suppressing the activity of sirtuin family proteins, which may affect lipid metabolism in liver cells. However, it is not clear how long-term ingestion of ethanol eventually causes lipid accumulation in liver. Here, we demonstrate that chronic ethanol ingestion activates peroxisome proliferator-activated receptor $\gamma$ (PPAR $\gamma$ ) and its target gene, monoacylglycerol O-acyltransferase 1 (MGAT1). During ethanol metabolism, a low NAD ${ }^{+}$NADH ratio repressed NAD-dependent deacetylase sirtuin 1 (SIRT1) activity, concomitantly resulting in increased acetylated PPAR $\gamma$ with high transcriptional activity. Accordingly, SIRT1 transgenic mice exhibited a low level of acetylated PPAR $\gamma$ and were protected from hepatic steatosis driven by alcohol or PPAR $\gamma 2$ overexpression, suggesting that ethanol metabolism causes lipid accumulation through activation of PPAR $\gamma$ through acetylation. Among the genes induced by PPAR $\gamma$ upon alcohol consumption, MGAT1 has been shown to be involved in triglyceride synthesis. Thus, we tested the effect of MGAT1 knockdown in mice following ethanol consumption, and found a significant reduction in alcohol-induced hepatic lipid accumulation. These results suggest that MGAT1 may afford a promising approach to the treatment of fatty liver disease.

Alcoholic liver disease, which is a major cause of morbidity and mortality worldwide, is associated with increased cardiovascular disease and diabetes ${ }^{1}$. Accumulation of fat in the liver in response to alcohol consumption can lead to more harmful forms of liver disease such as fibrosis, cirrhosis, and end-stage liver injury. Despite numerous studies on the pathogenesis of alcoholic liver diseases, targeted therapies based on the mechanism by which alcohol consumption causes hepatic steatosis are unavailable. The spectrum of alcoholic liver disease ranges from simple steatosis to more serious injury including cirrhosis. Because fatty liver damage is reversible when detected early, the best way to resolve this alcohol-mediated liver damage at this stage is abstaining from alcohol ${ }^{2}$. However, in order to provide improved, more effective therapies, elucidation of the mechanism by which ethanol metabolism causes lipid accumulation in the liver is required.

Recent studies indicate that ethanol increases fatty acid synthesis in hepatocytes by regulating lipid metabolism-associated transcription factors such as sterol regulatory element-binding protein 1c (SREBP1c) and carbohydrate-responsive element-binding protein (ChREBP), which promote fatty acid synthesis via up-regulation of lipogenic genes ${ }^{3}$. While ethanol is metabolized in liver, alcohol dehydrogenase and aldehydrogenase catalyze the conversion of $\mathrm{NAD}^{+}$to $\mathrm{NADH}$, thereby reducing the $\mathrm{NAD}^{+} / \mathrm{NADH}$ ratio similar to the fed state, in which the glycolysis pathway is activated and, in turn, sirtuin 1 (SIRT1) is inactivated ${ }^{4}$. SIRT1 is an NAD-dependent deacetylase that is activated in response to fasting, caloric restriction, and physical exercise ${ }^{5}$.

${ }^{1}$ Department of Biochemistry and Molecular Biology, Integrated Genomic Research Center for Metabolic Regulation, Institute of Genetic Science, Yonsei University College of Medicine, Seoul 120-752, Korea. ${ }^{2}$ Brain Korea 21 PLUS Project for Medical Science, Yonsei University, Seoul 120-752, Korea. ${ }^{3}$ Division of Metabolic Disease, Center for Biomedical Sciences, National Institutes of Health, Cheongwon-gun, Chungbuk 363-951, Korea. ${ }^{4}$ Department of Internal Medicine, Yonsei University College of Medicine, Seoul 120-752, Korea. ${ }^{5}$ Severance Biomedical Science Institute, Yonsei University College of Medicine, Seoul 120-752, Korea. Correspondence and requests for materials should be addressed to J.H.Y. (email: junghwan0081@yuhs.ac) or J.-W.K. (email: japol13@yuhs.ac) 
This enzyme plays an important role in hepatic lipid metabolism by modulating the acetylation of transcription factors such as peroxisome proliferator-activated receptor gamma coactivator $1-\alpha(P G C-1 \alpha)^{6}$. Recent research reveals that SIRT1 signaling is also associated with alcoholic liver disease as SIRT1 stimulation protects against alcohol-induced liver damage $e^{6,7}$. Because ethanol metabolism mimics the fed state even in the absence of glucose in terms of the $\mathrm{NAD}^{+} / \mathrm{NADH}$ ratio, these studies suggest that ethanol metabolism affects lipid metabolism through SIRT1 activity.

In adipocytes, SIRT1 represses peroxisome proliferator-activated receptor $\gamma(\operatorname{PPAR} \gamma)$ activity by recruiting a co-repressor, which results in fat mobilization ${ }^{8}$. Others have reported that SIRT1 directly deacetylates PPAR $\gamma$ , promoting brown remodeling of white adipocytes ${ }^{9}$. Thus, one of the mechanisms by which SIRT1 modulates hepatic lipid metabolism may involve PPAR $\gamma$, a master regulator of lipid metabolism ${ }^{10,11}$. Among the two isoforms, PPAR $\gamma 2$ is mainly present in adipose tissue, intestines, and macrophages to regulate fatty acid storage and glucose metabolism. In normal liver, PPAR $\gamma$ expression remains low. However, its expression is increased in a mouse model of obesity and plays a critical role in hepatic steatosis by regulating the expression of lipogenic genes $^{12}$. In addition, hepatic PPAR $\gamma$ expression is associated with triglyceride (TG) synthesis and lipid accumulation $^{13,14}$. Recently, we reported that increased PPAR $\gamma 2$ expression is a major contributor to high-fat-diet-induced hepatic steatosis, demonstrating that monoacylglycerol O-acyltransferase 1 (MGAT1), a PPAR $\gamma$-regulated enzyme, plays a critical role in lipid accumulation ${ }^{15}$.

MGAT1 is an enzyme that catalyzes the synthesis of diacylglycerol from monoacylglycerol and fatty acyl CoA. Thus, MGAT1 contributes to lipid accumulation via an alternative pathway for TG synthesis ${ }^{16}$. MGAT1, along with PPAR $\gamma$, is expressed at low levels in normal liver but is highly up-regulated in diet-induced hepatic steatosis $^{15}$. In this regard, inhibiting MGAT1 expression eventually suppressed hepatic lipid accumulation, as demonstrated by several studies in which MGAT1 is knocked down by adenovirus-mediated small hairpin RNA $(\operatorname{shRNA})^{15}$, antisense oligonucleotides ${ }^{17}$, and liver-specific non-viral small interfering RNA (siRNA $)^{18}$. Although these studies are restricted to non-alcoholic hepatic steatosis, we hypothesize that PPAR $\gamma$ and its downstream effector MGAT1 may play a role in alcohol-induced hepatic lipid accumulation if SIRT1 affects hepatic PPAR $\gamma$ during ethanol metabolism.

In this study, we found that PPAR $\gamma$ is activated in alcohol-induced hepatic steatosis and associated with reducing SIRT1 activity upon ethanol metabolism. Furthermore, our data suggest that inhibition of MGAT1 efficiently attenuated lipid accumulation due to alcohol consumption in liver. Therefore, we suggest that PPAR $\gamma$ is an important regulator of ethanol-induced hepatic steatosis, and that the development of an MGAT1 inhibitor could be an effective therapy for treating alcoholic or non-alcoholic hepatic steatosis.

\section{Results}

Ethanol decreased the NAD+/NADH ratio and SIRT1 activity. To study the effects of alcohol metabolism, we used C57BL/6 (B6) mice, which are widely used in metabolic disease research. Male B6 mice were pair-fed a Liber-Decarli liquid diet with or without (27\% of total calories) ethanol for 4 weeks (Fig. 1a). Exposure to an ethanol diet for 4 weeks resulted in a significant increase in hepatic TG content and liver weight, indicating that ethanol-induced hepatic steatosis and increased liver weight (Fig. 1b,c; oil-red-O staining shown in Supplementary Fig. S1). Notably, the $\mathrm{NAD}^{+} / \mathrm{NADH}$ ratio was significantly decreased in ethanol-fed mice (Fig. 1c). The level of SIRT1, an NAD-dependent deacetylase, also decreased, while PPAR $\gamma$ was not remarkably changed (Fig. 1d). However, acetylation of PPAR $\gamma$ clearly increased in ethanol-fed mice (Fig. 1e), suggesting that the SIRT1 activity required to deacetylate PPAR $\gamma$ is suppressed upon ethanol feeding. Increased PPAR $\gamma$ acetylation resulted in marked up-regulation of its target genes, including MGAT1, CD36, and G0s2, while PPAR $\gamma$ mRNA increased slightly (Fig. 1f). Another transcription factor known to play an important role in the pathophysiology of alcoholic hepatic steatosis, SREBP1c, was also highly expressed along with its target genes, which include liver-type pyruvate kinase (L-PK), stearyl CoA desaturase 1 (SCD1), glycerol phosphate acyltransferase (GPAT), fatty acid elongase 6 (Elvol6), and fatty acid synthase (FAS) (Fig. 1f). These data indicate that the fatty acid de novo synthesis pathway is also elevated following alcohol ingestion.

The mRNA levels of the seven known types of sirtuins revealed that expression of all sirtuin types was reduced in the alcohol-fed group (Supplementary Fig. S2). Of these, we checked the relationship between SIRT1 and ethanol-induced lipid metabolism, mainly focused on PPAR $\gamma$ activity. Further studies may be required to explore the linkage between alcohol metabolism and other sirtuins such as sirtuin 3 and 6, which are known to be involved in body metabolism.

SIRT1 protects the liver from alcohol-induced hepatic steatosis. Next, we used SIRT1 transgenic mice to evaluate its role in ethanol metabolism. Wildtype and SIRT1 transgenic mice were pair-fed a Liber-Decarli liquid diet with ethanol ( $27 \%$ of total calories) for 4 weeks. While wildtype mice developed a significant accumulation of lipid in the liver, SIRT1 transgenic mice displayed a protective effect on ethanol-induced hepatic steatosis (Fig. 2a; oil-red-O staining shown in Supplementary Fig. S1). Throughout the experimental period, food intake was similar and ethanol feeding had no apparent effect on the health status of both mouse groups. Although body weight remained unchanged, the liver weights were lower in SIRT1 transgenic mice (Fig. 2b). Liver TG content in these mice was also decreased significantly compared to wildtype animals (Fig. 2b). The level of PPAR $\gamma$ protein did not differ significantly in SIRT1 transgenic mice (Fig. 2c), but acetylation of PPAR $\gamma$ appeared to decrease (Fig. 2d). Furthermore, the expression of PPAR $\gamma$ and its target genes, including MGAT1, aP2/422, and CD36, was also reduced (Fig. 2e). These data suggest that PPAR $\gamma$ activity, with subsequent PPAR $\gamma$ expression, also decreased. The levels of aspartate transaminase (AST) and alanine transaminase (ALT), which are indicators of liver inflammation, were also decreased in SIRT1 transgenic mice (Fig. 2f). 


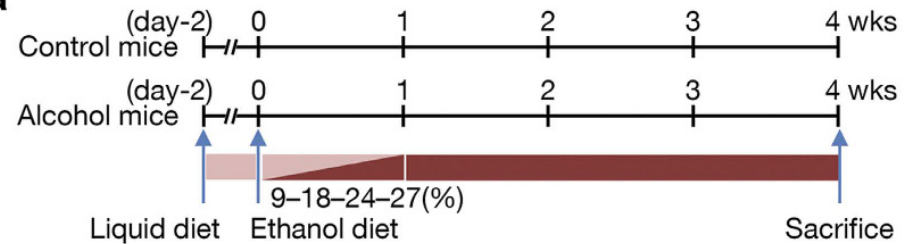

b

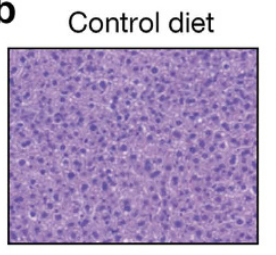

Ethanol diet

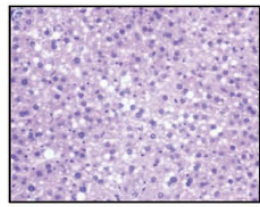

C
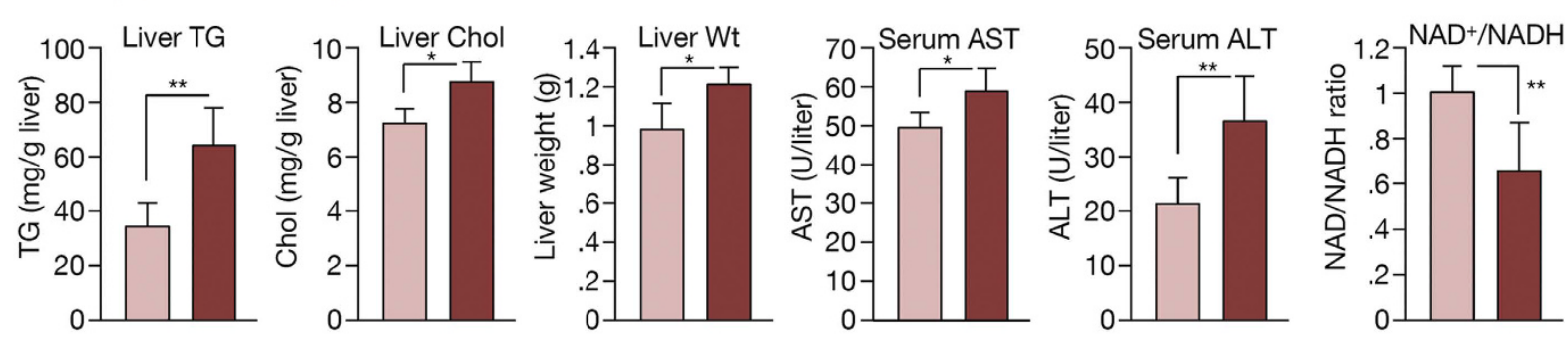

d

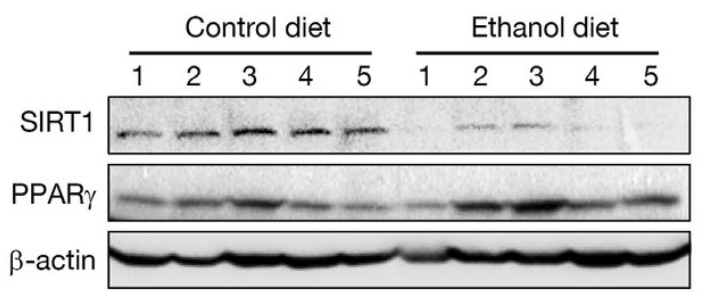

e
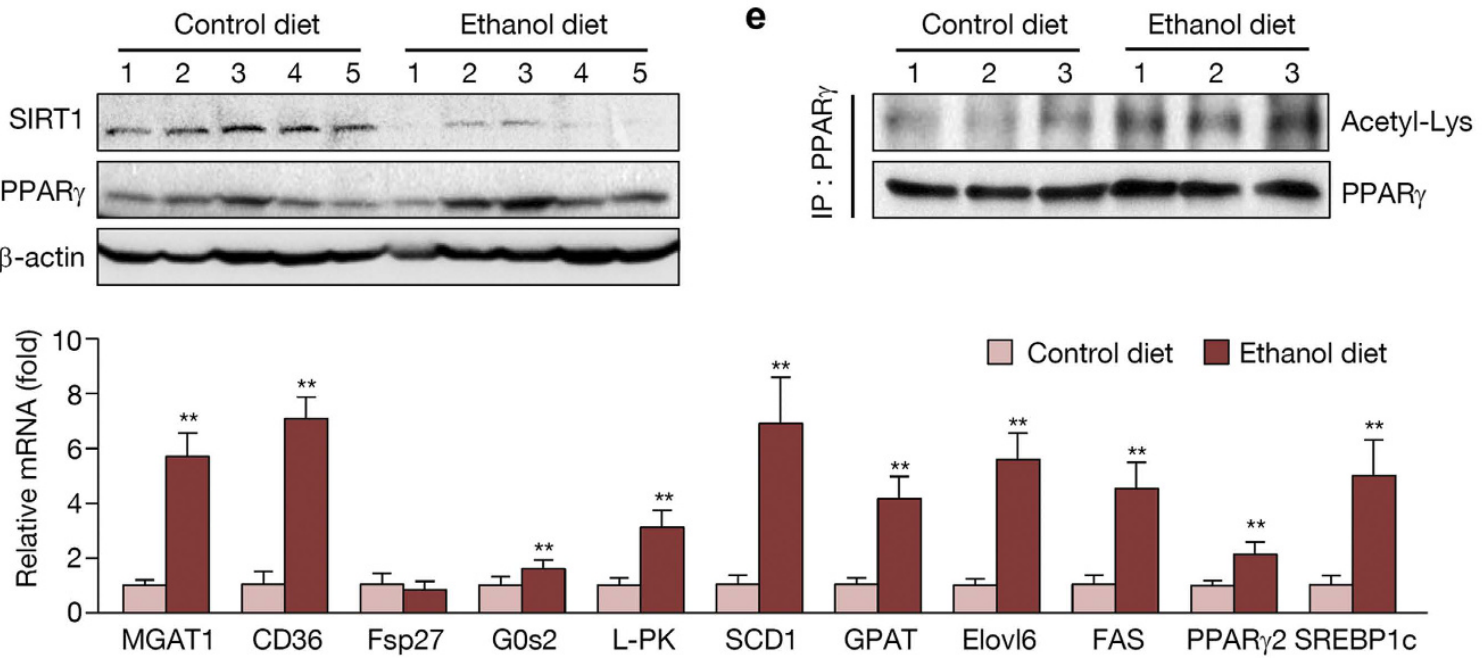

Figure 1. Ethanol decreases the NAD ${ }^{+} / \mathrm{NADH}$ ratio and SIRT1 activity to cause PPAR $\gamma$ acetylation.

(a) Schedule of ethanol and control diet regimens. (b) H\&E staining performed on liver sections from mice.

(c) Hepatic TG and cholesterol content, liver weight, serum aspartate transaminase (AST) alanine transaminase (ALT) levels, and $\mathrm{NAD}^{+} / \mathrm{NADH}$ ratio in the livers of control diet and ethanol diet-fed mice. ( $\mathrm{n}=6$ per group)

(d) Western blot analysis of SIRT1, PPAR $\gamma$, and $\beta$-actin. ( $\mathrm{n}=5$ per group) (e) PPAR $\gamma$ acetylation in control and ethanol diet-fed mice. ( $\mathrm{n}=3$ per group) (f) Expression of PPAR $\gamma$ and SREBP1c and their target genes in control and ethanol diet-fed mice. ( $\mathrm{n}=6-8$ per group) Data represent the mean \pm SD. $* \mathrm{P}<0.05, * * \mathrm{P}<0.01$.

Because these transgenic mice express SIRT1 throughout the body, we could not rule out effects from other organs such as adipose tissue. To examine the effect of SIRT1 on hepatic lipid accumulation directly, we employed adenoviruses as vectors to express SIRT1 because they preferentially target the liver in mice ${ }^{19}$. During ethanol feeding, adenovirus overexpressing GFP or SIRT1 was injected into the tail vein 2 weeks before the end of the experimental period (Fig. 3a). This administration led to robust expression of hepatic SIRT1 (Fig. 3b), which attenuated ethanol-induced hepatic lipid accumulation (Fig. 3c,d). Interestingly, liver weight was similar with this short-term expression of SIRT1 (Fig. 3d). SIRT1 overexpression in the liver caused down-regulation of PPAR $\gamma$ and its target gene, MGAT1 (Fig. 3e), suggesting that ethanol metabolism eventually affects lipid metabolism through SIRT1. Taken together, these data indicate that SIRT1 has a protective and beneficial role in alcohol-induced hepatic steatosis.

SIRT1 attenuates PPAR $\gamma 2$-induced hepatic steatosis. We demonstrated that SIRT1 overexpression attenuates ethanol-induced hepatic steatosis, in which PPAR $\gamma$ acetylation is decreased. To verify the involvement of PPAR $\gamma$ in SIRT1-mediated effects on the fatty liver, we administered adenoviral PPAR $\gamma 2$ (Ad-PPAR $\gamma 2$ ) into wildtype or SIRT1 transgenic mice via tail vein injection (Fig. 4a). As reported previously ${ }^{15}$, Ad-PPAR $\gamma 2$ injection produced severe hepatic steatosis (Fig. 4b; oil-red-O staining shown in Supplementary Fig. S1) with higher levels of hepatic TG than control Ad-GFP mice (Fig. 4c). Histological analysis revealed the presence of numerous fat droplets in the liver of Ad-PPAR $\gamma 2$-injected mice. However, SIRT1 transgenic mice overexpressing PPAR $\gamma 2$ exhibited lower levels of hepatic TG than wildtype mice (Fig. 4b,c). Ad-PPAR $\gamma 2$ injection induced the expression 
a

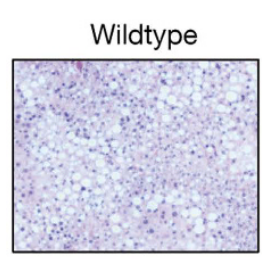

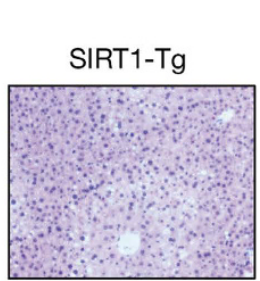

b

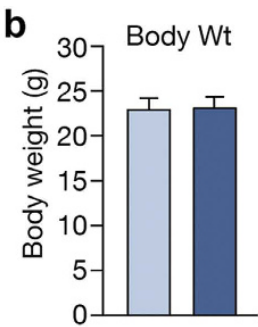

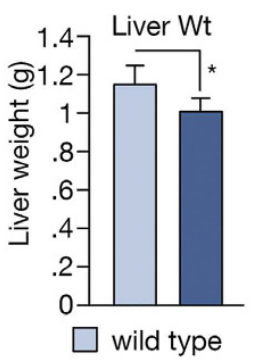
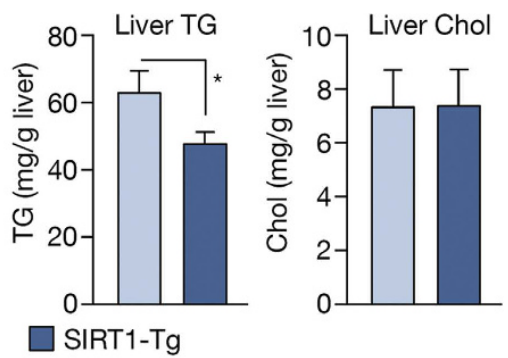

C

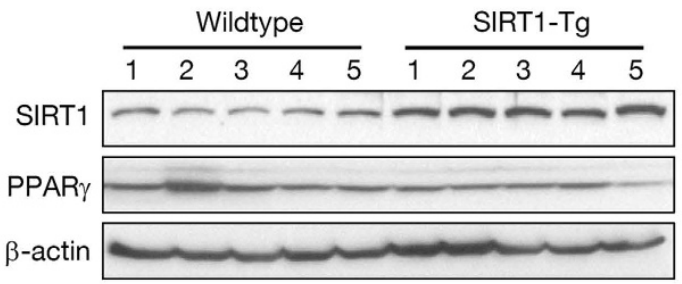

d

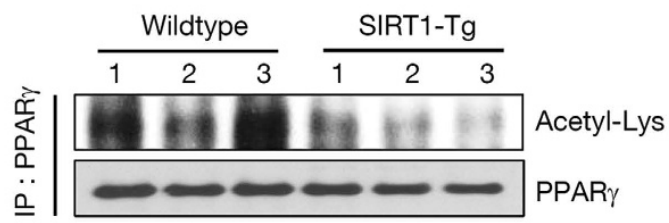

e

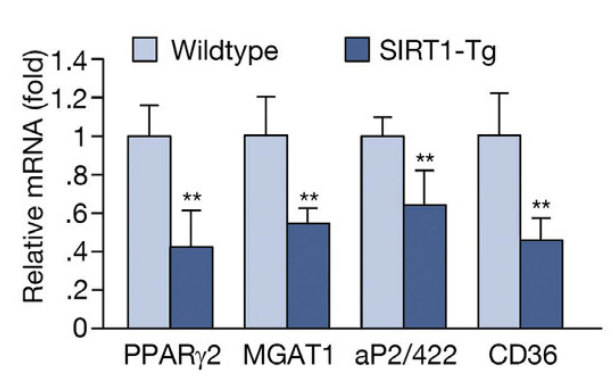

f

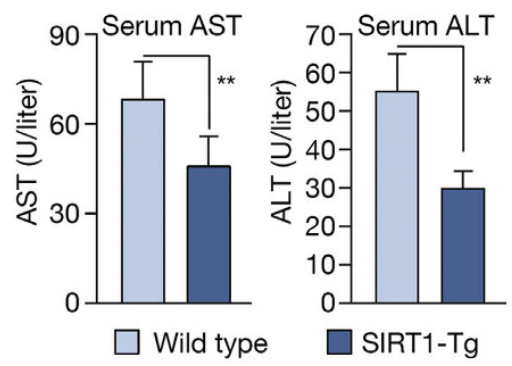

Figure 2. SIRT1 transgenic mice exert a protective effect on alcohol-induced hepatic steatosis. (a) H\&E staining performed on liver sections from mice. (b) Body weight, liver weight, hepatic TG, and cholesterol in wild type and SIRT1 transgenic mice. (c) Western blot analysis of SIRT1, PPAR $\gamma$, and $\beta$-actin. ( $\mathrm{n}=5$ per group) (d) PPAR $\gamma$ acetylation in wild type and SIRT1 transgenic mice. $(\mathrm{n}=3$ per group) (e) Real-time PCR analysis of PPAR $\gamma$ and its target genes in liver. (f) AST and ALT levels in blood samples. ( $n=6$ per group). Data represent the mean $\pm \mathrm{SD}$. $* \mathrm{P}<0.05$, **P $<0.01$.

a

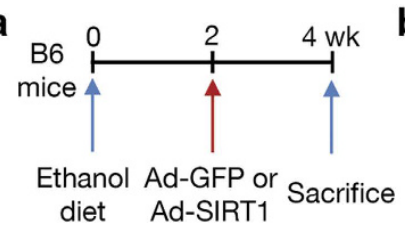

d

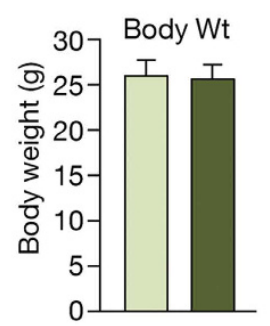

b

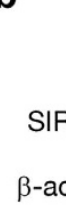

C

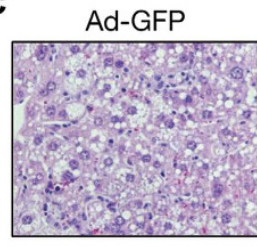

e

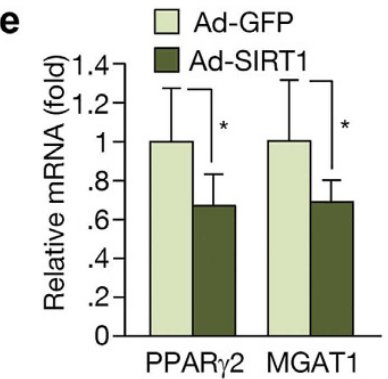

Figure 3. The effect of SIRT1 overexpression via adenovirus injection on alcohol-induced hepatic steatosis. (a) Schedule of SIRT1 adenovirus injection. Ad-SIRT1 or Ad-GFP was introduced via tail vein injection at 2 weeks after initiation of ethanol feeding. The mice were sacrificed 2 weeks later. (b) Western blot analysis showing SIRT1 expression in the liver of Ad-GFP- and Ad-SIRT1-injected mice. (c) H\&E staining performed on liver sections from mice. (d) Body weight, liver weight, hepatic TG, and cholesterol content. (e) Real-time PCR analysis of PPAR $\gamma$ and MGAT1 expression. $(n=5)$ Data represent the mean \pm SD. $* P<0.05$. 


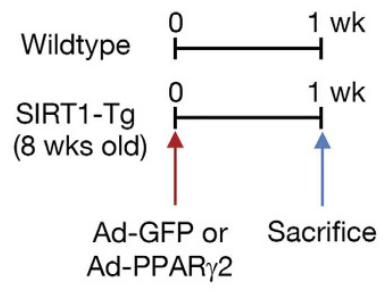

C

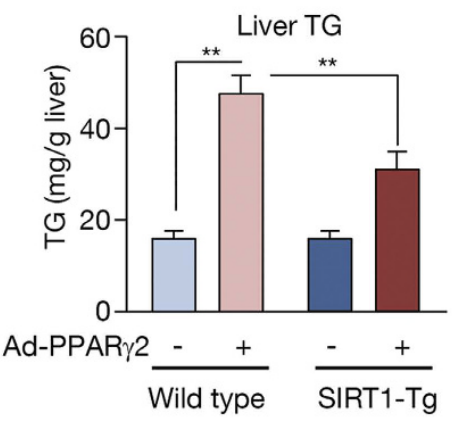

b

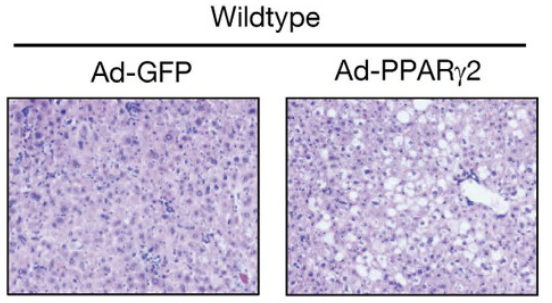

d

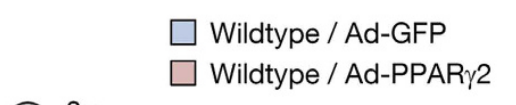

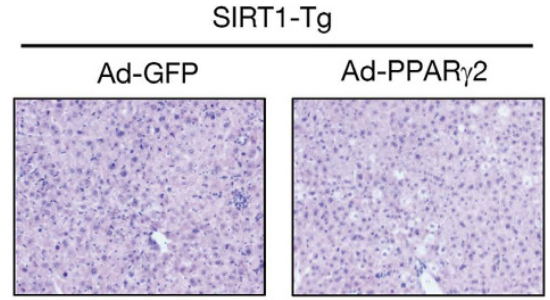

SIRT1-Tg / Ad-GFP

SIRT1-Tg / Ad-PPAR 2

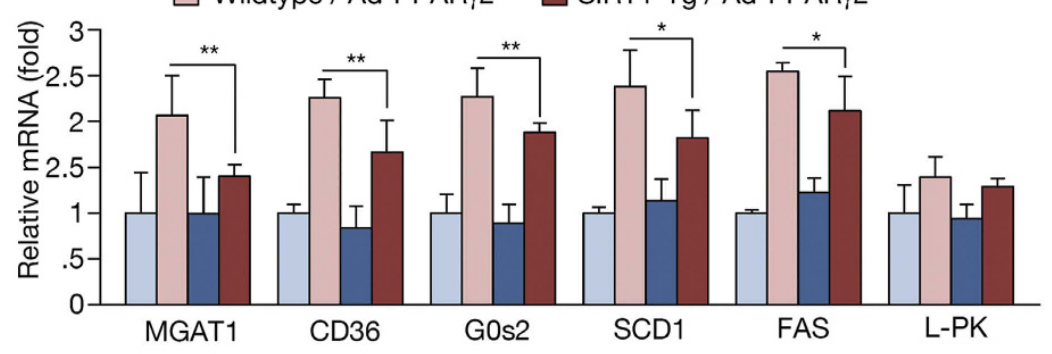

e

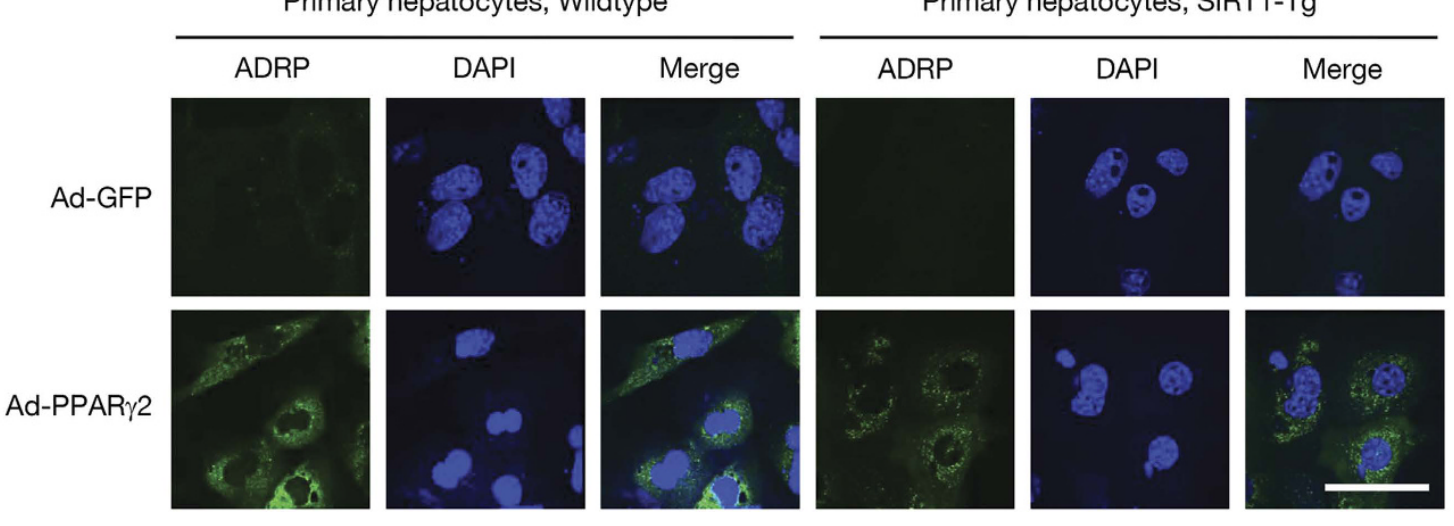

Figure 4. The effect of PPAR $\gamma 2$ overexpression on wild type and SIRT1 transgenic mice. (a) Schedule of PPAR $\gamma 2$ overexpression. Wild type and SIRT1 transgenic mice were injected with Ad-PPAR $\gamma 2$ or an Ad-GFP via the tail vein, fed a chow diet for 1 week, and then sacrificed. (b) H\&E staining performed on liver sections of wild type and SIRT1 transgenic mice. (c) Hepatic TG content in wild type and SIRT1 transgenic mice infected with Ad-GFP or Ad-PPAR $\gamma 2$. (d) Real-time PCR analysis of PPAR $\gamma$ target genes and lipogenic genes in wild type and SIRT1 transgenic mice. (e) Immunofluorescence staining for adipose differentiation-related protein (ADRP, green) in mouse primary hepatocytes. Nuclei were stained with DAPI and fluorescence was visualized by confocal microscopy. Scale bar $=50 \mu \mathrm{m}$. Data in $\mathrm{c}$ and $\mathrm{d}$ represent the mean $\pm \mathrm{SD}$. $* \mathrm{P}<0.05, * * \mathrm{P}<0.01$.

of several PPAR $\gamma$ targets and lipogenic genes. However, this increase in gene expression was attenuated in SIRT1 transgenic mice (Fig. 4d).

We also analyzed the effect of PPAR $\gamma 2$ overexpression in primary mouse hepatocytes. These cells were subjected to immunocytochemistry to detect adipose differentiation-related protein (ADRP), which is a target gene of PPAR $\gamma$ that localizes to the lipid droplet. PPAR $\gamma 2$ overexpression resulted in increased ADRP expression in primary hepatocytes; however, this increase was significantly attenuated in cells isolated from SIRT1 transgenic mice (Fig. 4e). These results suggest that PPAR $\gamma$ acts as a major regulator in hepatic TG synthesis and SIRT1 inhibits PPAR $\gamma$-induced hepatic steatosis.

MGAT1 knockdown protects against alcohol-induced hepatic steatosis. Previously, we reported that knockdown of PPAR $\gamma$-regulated MGAT1 expression can successfully improve diet-induced non-alcoholic hepatic steatosis in murine and human models ${ }^{15,20}$. Our results shown here strongly suggest that the PPAR $\gamma$-MGAT1 axis contributes to the development and aggravation of lipid accumulation both in non-alcoholic and alcoholic hepatic steatosis. However, the mechanism of PPAR $\gamma$ activation might be different. To examine whether MGAT1 suppression can reduce ethanol-induced hepatic steatosis, male B6 mice were fed a Liber-Decarli liquid diet with or without ethanol (27\% of total calories) for 3 weeks and then administered with adenoviral sh-control or sh-MGAT1 via tail vein injection. After 1 week of continued diet, mice were sacrificed for analysis (Fig. 5a). As shown in Fig. 5b (oil-red-O staining shown in Supplementary Fig. S1), knockdown of 


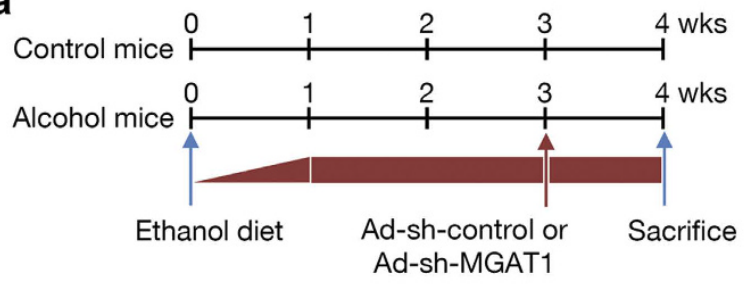

C
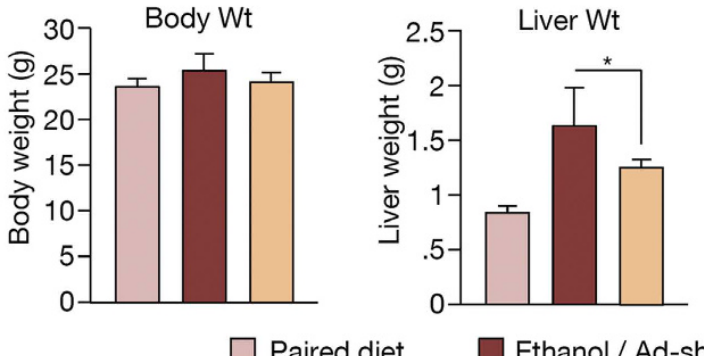

b

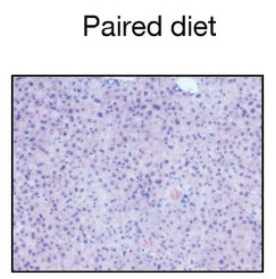

Ethanol diet

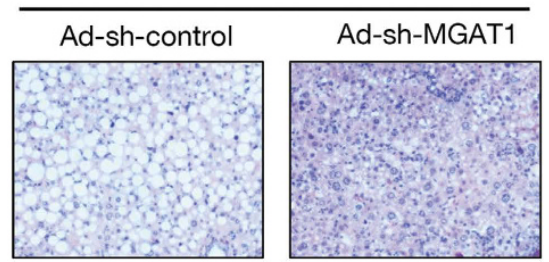

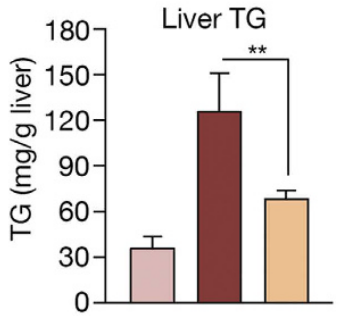

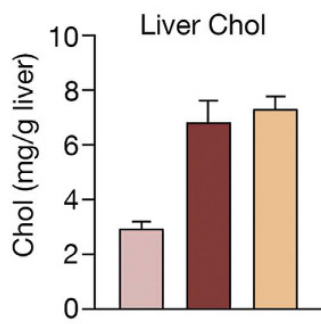

Ethanol / Ad-sh-control

Ethanol / Ad-sh-MGAT1

d

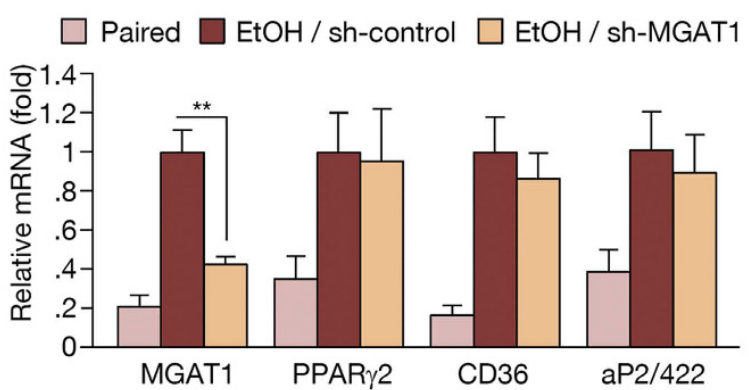

e

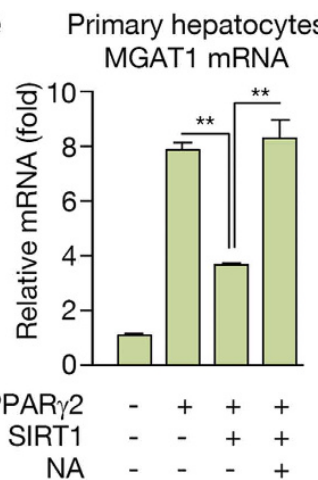

f

HepG2 cells Luciferase

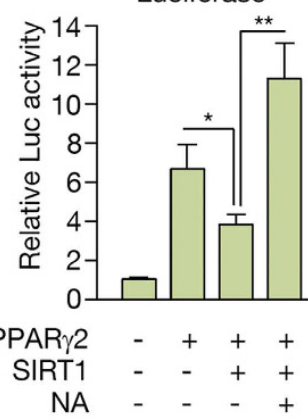

Figure 5. MGAT1 knockdown suppresses alcohol-induced hepatic steatosis. (a) Schedule of MGAT1 knockdown in ethanol-fed mice. Adenoviral sh-control or sh-MGAT1 was introduced via tail vein injection at 3 weeks after initiation of ethanol feeding. One week later, the mice were sacrificed. (b) H\&E staining performed on liver sections from mice in paired, ethanol-fed with sh-control, and ethanol-fed with sh-MGAT1 groups. (c) Body weight, liver weight, hepatic TG, and cholesterol content. (d) Real-time PCR analysis showing the expression of PPAR $\gamma$ and its target genes. $(n=6)$. Data represent the mean \pm SD. $* P<0.05$. (e) Primary hepatocytes $\left(4 \times 10^{5}\right.$ cells per well) cultured on 6-well plates were infected with 50 MOI of Ad-GFP, Ad-PPAR $\gamma$ 2, and Ad-SIRT1 with or without nicotinamide (NA, $10 \mu \mathrm{M})$. MGAT1 mRNA was analyzed by real-time PCR. (f) Luciferase assay using human MGAT1 promoters ( $2 \mathrm{~kb})$ was performed. In HepG2 cells, MGAT1 promoter constructs were co-transfected with or without PPAR $\gamma 2 / R X R \alpha$ overexpression vectors along with $100 \mathrm{MOI}$ of either Ad-GFP or Ad-SIRT1. Data represent the mean \pm SD from three independent experiments performed in duplicate. $* \mathrm{P}<0.05, * * \mathrm{P}<0.01$.

hepatic MGAT1 significantly improved ethanol-induced hepatic steatosis. The hepatic TG level was reduced by $42 \%$ after only 1 week of MGAT1 knockdown (Fig. 5c). Furthermore, hepatic MGAT1 knockdown also resulted in decreased liver weight, suggesting that MGAT1 expression plays an important role in alcohol-induced hepatic steatosis (Fig. 5c). Targeting MGAT1 did not affect the expression of PPAR $\gamma$ and its other regulatory genes such as aP2/422 and CD36, suggesting that MGAT1 is a critical factor involved in the accumulation of hepatic lipid upon ethanol feeding (Fig. 5d).

To verify the role of the SIRT1-PPAR $\gamma$-MGAT1 axis in this process, we tested MGAT1 mRNA expression in the presence of PPAR $\gamma$ and/or SIRT1. As shown in Fig. 5e, SIRT1 efficiently suppressed PPAR $\gamma 2$-induced MGAT1 expression. Nicotinamide (NA), a known SIRT1 inhibitor, counteracted the suppressive effects of SIRT1. Luciferase assays also showed that SIRT1 suppresses PPAR $\gamma 2$-induced MGAT1 promoter activity in human hepatoma-derived HepG2 cells (Fig. 5f). To evaluate whether SREBP1c, another major transcription regulator of hepatic steatosis, regulates MGAT expression, we overexpressed SREBP1c into human HepG2 cells. Our data show that SREBP1c did not affect the expression level of all three MGAT subtypes in human cells (Supplementary Fig. S3), suggesting that increased SREBP1C and PPAR $\gamma$ during ethanol feeding contributes to lipid accumulation via distinct mechanisms.

A previous study reported that PPAR $\gamma$ acetylation sites (lysine residues 98, 107, 218, 268, and 293) were affected by SIRT1 ${ }^{9}$. Therefore, we generated various PPAR $\gamma 2$ mutant forms (K98R, K107R, K218R, K268R, and 
K293R) to evaluate the role of the acetylation sites in modulating PPAR $\gamma$-induced MGAT1 expression. However, as shown in Supplementary Fig. S4, we could not find a significant difference in PPAR $\gamma$ activity following mutation of the acetylation sites, which regulate MGAT1 expression. This result suggests that MGAT1 expression is regulated by acetylation on other sites or combination of various acetylation.

Ethanol metabolism in binge drinking. Binge drinking is the act of consuming heavy amounts of alcohol in a short time period. Recently, the role of ChREBP in binge drinking was reported ${ }^{3}$. In this report, acute alcohol consumption affected the activity of ChREBP by regulating its acetylation. Based on this finding, we explored the role of PPAR $\gamma$ and the early response to alcohol consumption through a binge drinking mouse model. Twelve-week-old male C57BL/6J mice were fasted for $4 \mathrm{~h}$ before receiving two doses of gavages with equivalent calories of DM or ethanol at $3.5 \mathrm{~g} / \mathrm{kg}$ (Fig. 6a). Examination of these animals revealed that the $\mathrm{NAD}^{+} /$ $\mathrm{NADH}$ ratio was decreased in the binge drinking group compared to the DM group (Fig. 6b). The SIRT1 level was also decreased after binge drinking (Fig. 6c). PPAR $\gamma$ expression and its acetylation were increased with binge drinking (Fig. 6d,e). Also, the level of SREBP1c and ChREBP mRNA was increased in the livers of the ethanol-consuming group (Fig. 6e). Accordingly, the expression of SREBP1c and ChREBP target genes such as SCD1, L-PK, and FAS increased; however, expression of PPAR $\gamma$ target genes remained at a low level (Fig. 6f). These results suggest that the expression level of PPAR $\gamma$ in this setting was not sufficient to induce its target genes even though PPAR $\gamma$ mRNA was up-regulated by two-fold. Thus, we hypothesize that binge drinking initially affects SREBP 1 c or ChREBP to develop a mild steatosis primarily by fatty acid de novo synthesis, which later induces a sufficient level of acetylated PPAR $\gamma$ and its target genes, and ultimately aggravating hepatic steatosis (Fig. 7).

\section{Discussion}

Excessive alcohol consumption is an important public health problem that contributes substantially to the global burden of mortality and morbidity. In the 2010 Global Burden of Disease study, alcohol-attributable liver disease was responsible for 493,300 deaths (156,900 females and 336,400 males) representing $0.9 \%$ of all global deaths ${ }^{21}$. Therefore, efforts to reduce alcohol consumption and prevent alcohol-induced hepatic damage are needed. Alcoholic liver disease starts with hepatic steatosis, which is characterized by an increase in intrahepatic TG. Continuous alcohol consumption leads to hepatic inflammation and liver fibrosis. Studies estimate that a third of patients with steatosis will develop hepatic inflammation and $8 \%$ to $20 \%$ of patients with steatosis will eventually progress to cirrhosis ${ }^{1}$. The main causes for alcoholic fatty liver are known to be accumulation of acetaldehyde, tumor necrosis factor $\alpha$, endoplasmic reticulum stress, 2-arachidonoylgycerol, and adenosine, which increase SREBP1c activity and induce fatty acid synthesis, leading to alcoholic fatty liver. Other transcription factors also contribute to the pathogenesis of alcoholic liver disease. For example, PPAR $\alpha$, another major regulator, decreases fatty acid $\beta$-oxidation ${ }^{1}$. Recently, ChREBP was reported to play a role during the early period of alcohol consumption $^{3}$. On the other hand, hepatic PPAR $\gamma$, which is known as a critical regulator of TG accumulation in adipose tissue, has yet to be investigated in alcohol-induced hepatic steatosis until now.

A ligand-activated transcription factor, PPAR $\gamma$ is a member of the nuclear receptor superfamily that primarily regulates adipogenesis and lipid accumulation ${ }^{22}$. Because PPAR $\gamma$ expression in the liver is low compared to that in adipose tissue, the role of this transcription factor in the liver remains controversial ${ }^{13}$. In the past decade, studies found that hepatic PPAR $\gamma$ is significantly increased in an obese animal model and plays an important role in fatty liver formation ${ }^{23,24}$. In our study, alcohol-fed mice appeared to exhibit an increase in hepatic PPAR $\gamma$ protein to a certain extent; however, comparison to control mice nullified any apparent increase. However, the expression of PPAR $\gamma$ target genes increased in alcohol-fed mice. Therefore, we investigated the post-translational modification of PPAR $\gamma$ to examine how ethanol regulates PPAR $\gamma$ activity. Studies have demonstrated that PPAR $\gamma$ function is regulated by several post-translational modifications, including phosphorylation, acetylation, sumoylation, and ubiquitination ${ }^{24,25}$. PPAR $\gamma$ is phosphorylated within the AF1 region by mitogen-activated protein kinases or cyclin-dependent kinases (Cdk7 and Cdk9), which regulate PPAR $\gamma$ activity in opposing manners ${ }^{26}$. Moreover, it was found that PPAR $\gamma$ is phosphorylated within the ligand binding domain at Ser273 by Cdk 5 to control the expression of a distinct group of genes that becomes deregulated in obesity ${ }^{27}$. Sumoylation of PPAR $\gamma$ in the AF1 region represses its transcriptional activity, possibly by recruiting a co-repressor ${ }^{28}$. Additionally, PPAR $\gamma$ has been shown to be ubiquitinated, which is enhanced by ligand binding like thiazolidinediones ${ }^{29}$. The regulation of PPAR $\gamma$ function by acetylation has not been extensively studied; however, it is evident that PPAR $\gamma$ activity is associated with acetylation as PPAR $\gamma$ activity is attenuated by the presence of a deacetylase such as HDAC3 and SIRT $1^{30}$.

SIRT1 regulates lipid metabolism by deacetylating lysine residues on transcription factors such as SREBP1c and PGC1 $\alpha$ in liver ${ }^{31,32}$. However, the mechanism by which SIRT1 regulates hepatic PPAR $\gamma$ function, especially in alcoholic fatty liver, remains poorly understood. In this study, we established that SIRT1 is involved in alcoholic hepatic steatosis by regulating the acetylation of PPAR $\gamma$ and its activity. We also observed that PPAR $\gamma$ overexpression could induce hepatic steatosis, and that knockdown of MGAT1, a PPAR $\gamma$ target gene, could inhibit hepatic lipid accumulation. We previously showed that MGAT1 is a direct target gene of PPAR $\gamma$, by identifying functional PPAR-response element in the promoter ${ }^{15}$, and also reported that human MGAT1 is also regulated by PPAR $\gamma$ shown by luciferase assay ${ }^{20}$. Therefore, we propose that the PPAR $\gamma$ signaling pathway is critical in hepatic lipid synthesis and fatty liver formation. In order to examine whether other pathways may involve in the regulation of MGAT1 in ethanol feeding, we also investigated SREBP1c, which is reported to play a role in alcoholic hepatic steatosis. Supplementary Fig. S3, however, showed that the expression of MGAT genes did not change in response to SREBP1c overexpression. In addition, it is noteworthy that MGAT1 was not induced upon binge drinking shown in Fig. 6, where SREBP1c and ChREBP are highly expressed. Thus, it is unlikely that SREBP1c or ChREBP regulates MGAT1. Nevertheless, it is still possible that other pathways in ethanol feeding 
a

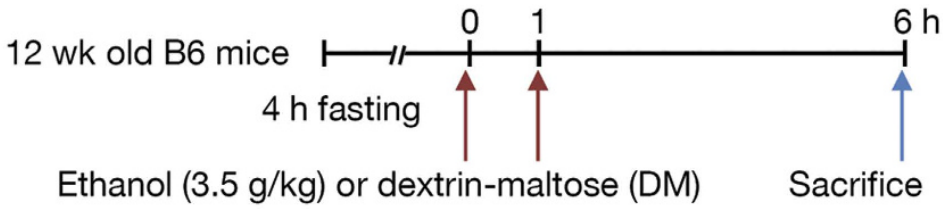

b

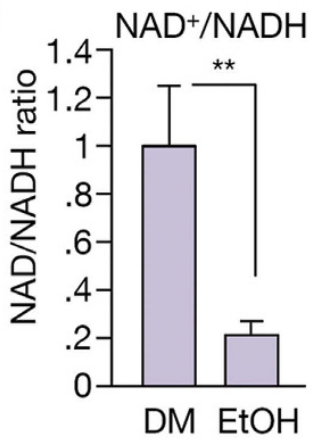

C

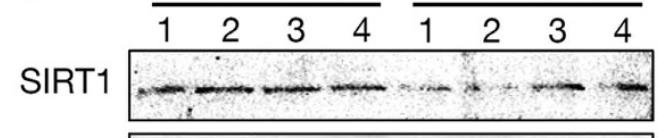

$\beta$-actin

d

I-Lys

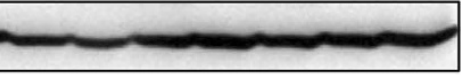

Acetyl-Lys
PPAR $\gamma$

e
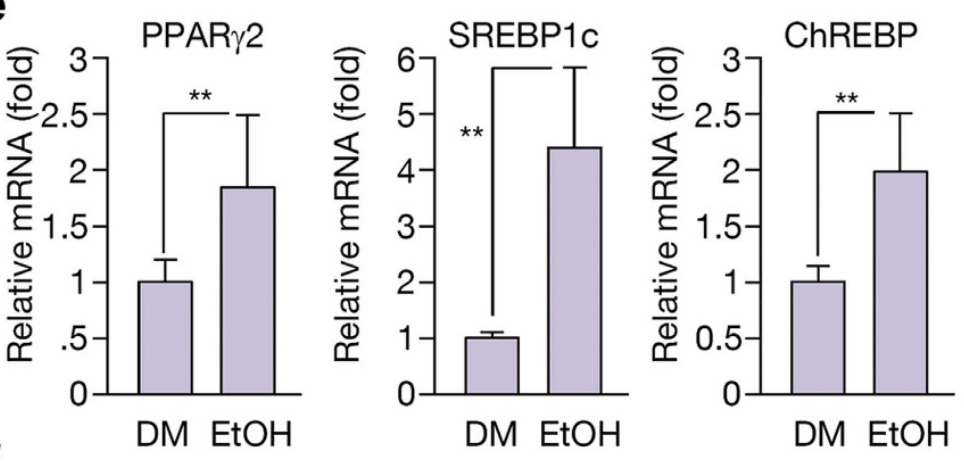

$\mathbf{f}$

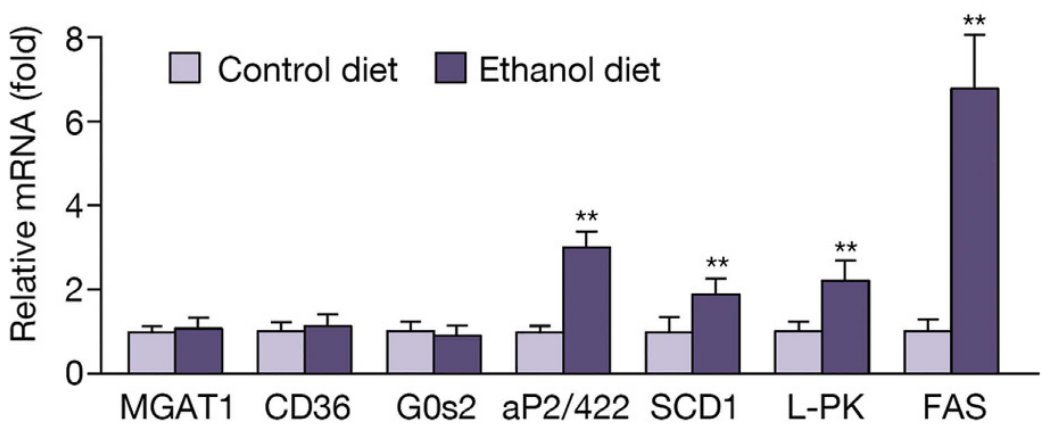

Figure 6. Ethanol metabolism in binge drinking leads to the activation of SREBP1c and ChREBP.

(a) Schedule of binge drinking experiment. B6 mice were fasted for $4 \mathrm{~h}$ before receiving two gavages of equivalent calories of ethanol at $3.5 \mathrm{~g} / \mathrm{kg}$ or dextrin-maltose $(\mathrm{DM})$. Mice were sacrificed $6 \mathrm{~h}$ later. (b) $\mathrm{NAD}^{+} / \mathrm{NADH}$ ratio examined in DM and ethanol-fed mice group. ( $\mathrm{n}=6$ per group) (c) Western blot analysis showing SIRT1 expression in DM and ethanol-fed mice group. ( $\mathrm{n}=4$ per group) (d) PPAR $\gamma$ acetylation in DM and ethanol-fed mice group. ( $\mathrm{n}=3$ per group) $(\mathbf{e}, \mathbf{f})$ Real-time PCR analysis showing expression of PPAR $\gamma$, SREBP1c, ChREBP, and their target genes. $(\mathrm{n}=6)$. Data represent the mean $\pm S D$. $* * P<0.01$.

regulate MGAT1, because the short-term binge drinking could not activate the MGAT1 transcription even in the induction of PPAR $\gamma$ and its acetylation (Fig. 6). It may be due to the lack of lipid signaling required for PPAR $\gamma$ transcriptional activity, but we cannot rule out the involvement of other pathways.

The causes of hepatic steatosis have been categorized as either alcoholic or non-alcoholic. Despite this, it is difficult to differentiate between these types of hepatic steatosis in the clinic. Furthermore, alcohol synergistically increases the prevalence and severity of hepatic steatosis in obese patients ${ }^{33,34}$. Discovering similar pathways between non-alcoholic and alcoholic hepatic steatosis is a useful strategy for developing effective therapeutics for treating hepatic steatosis. In this study, we identified that the SIRT1-PPAR $\gamma$-MGAT1 axis is critically involved in alcoholic hepatic steatosis, making MGAT1 a potential therapeutic target of hepatic fatty liver disease. 


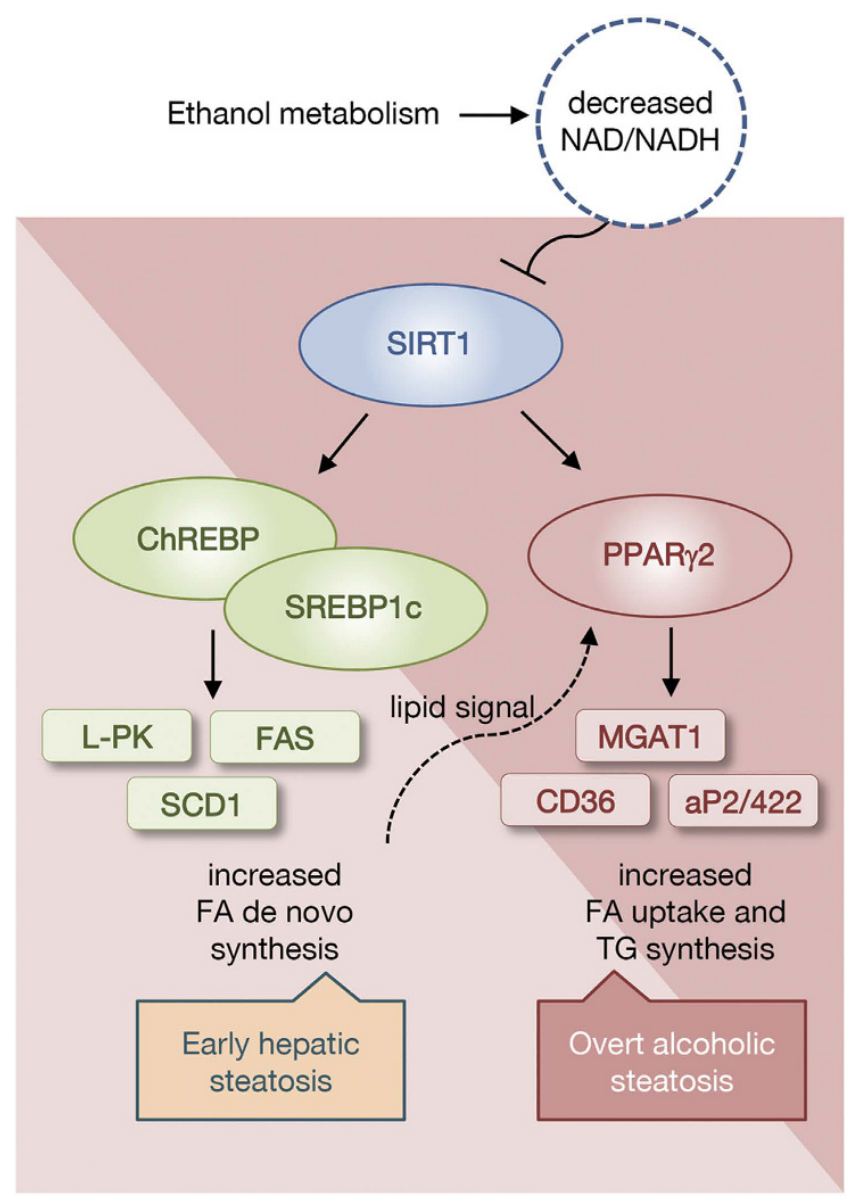

Figure 7. Model of alcohol-induced hepatic steatosis. During ethanol digestion, $\mathrm{NAD}^{+}$is converted into $\mathrm{NADH}$ by alcohol dehydrogenase and aldehyde dehydrogenase. A low $\mathrm{NAD}^{+} / \mathrm{NADH}$ ratio represses $\mathrm{NAD}$ dependent deacetylase sirtuin 1 (SIRT1) activity. Reduced SIRT1 activity causes the activation of SREBP1c, ChREBP, and PPAR $\gamma$. In the early phase, expression of SREBP1c and ChREBP target genes, such as L-PK, FAS, and SCD1, are increased, and then PPAR $\gamma$ target genes including MGAT1 are increased. Expression of PPAR $\gamma$ and its target genes lead to the development of alcoholic steatosis.

\section{Materials and Methods}

Mice and diet. Male C57BL/6J mice were purchased from SLC (Japan). The animals were maintained in a temperature-controlled room $\left(22^{\circ} \mathrm{C}\right)$ on a 12:12-h light-dark cycle. Mice (at 7 or 8 weeks of age) were fed a Lieber-DeCarli liquid diet (Dyets) containing $1 \mathrm{Kcal} / \mathrm{ml}$, of which $18 \%$ was derived from protein, $35 \%$ of fat, and either $47 \%$ from carbohydrate (control diet) or $20 \%$ from carbohydrate and $27 \%$ from ethanol (alcohol diet) for up to 4 weeks. Ethanol was introduced gradually by increasing the content by $9 \%$ of its total caloric-intake until the mice were consuming a diet containing $27 \%$ ethanol, which was then continued for three more weeks. Mice were paired-fed, and body weight and food intake were monitored daily. Body weight was measured once a week. Adenovirus injection $\left(2 \times 10^{9} \mathrm{pfu}\right)$ through the tail vein was administered at weeks 2 or 3 of the liquid diet period to suit each experiment. At the end of the experiment, mice were sacrificed and liver tissues and blood samples were collected. Male SIRT1 transgenic mice were purchased from Jackson Laboratory (Bar Harbor, ME). Binge drinking was modeled using a modified version of a recently published protocol ${ }^{3}$. Briefly, twelve-week-old male $\mathrm{C} 57 \mathrm{BL} / 6 \mathrm{~J}$ mice were fasted for $4 \mathrm{~h}$ before receiving two gavages of equivalent calories of ethanol at $3.5 \mathrm{~g} / \mathrm{kg}$ or dextrin-maltose (DM; MP Biomedicals, Santa Ana, CA). Mice were kept on a heating pad for the duration of the experiment to prevent hypothermia. Then, mice were sacrificed $6 \mathrm{~h}$ later. Liver tissues were fixed with $10 \%$ (vol/vol) formalin and embedded in paraffin, and were stained with H\&E or oil-red-O. All experimental protocols involving animals, including maintenance and care, were performed in accordance with the National Institutes of Health guidelines and ethics guidelines of Yonsei University, and all animal procedures were approved by the Committee on Animal Investigations of Yonsei University.

RNA isolation and analysis of gene expression by quantitative RT-PCR. Total RNA was isolated using TRIzol reagent (Invitrogen, Carlsbad, CA) according to the manufacturer's instructions. First-strand cDNA synthesis from $5 \mu \mathrm{g}$ total RNA was performed using SuperScript III reverse transcriptase (Invitrogen) primed with random hexamer primers. Real-time qPCR was performed using SYBR Green Master mix (Applied Biosystems) with a Step One instrument (Applied Biosystems, Foster City, CA). 
Expression of Rplp0 was also measured as an invariant control. The primer sequences used in real-time qPCR are as follows: PPAR $\gamma, 5^{\prime}$-CTCTGGGAGATTCTCCTGTT-3' 5' $^{\prime}$-GGTGGGCCAGAATGGCATCT-3'; SREBP1c, 5'-GGAGCCATGGATTGCACATT-3', 5'-GGCCCGGGAAGTCACTGT-3'; ChREBP, 5'-CCTC ACTTCACTGTGCCTCA-3', 5' -ACAGGGGTTGTTGTCTCTGG-3'; aP2/422, 5' -TCTCCAGTGAAAA CTTCGAT-3', 5'-TACGCTGATGATCATGTTG-3'; FSP27, 5'-TCCAGGACATCTTGAAACTT-3', 5'-GGCTTGCAAGTATTCTTCTG T-3'; Cd36, 5'-TGCACCACATATCTACCAAA-3' ' 5' -TTGTAACCCCAC

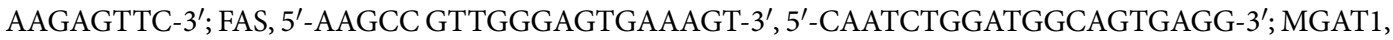
5'-CTGGTTCTGTTTCCCGTTGT-3', 5' -TGGGTCAAGGCCATCTTAAC-3'; L-PK, 5'-CCGAGATACGC ACTGGAGTC-3', 5'-GTGGTAGTCCACCCACACTG-3'; SCD1, 5'-TTCTCAGAAACACACGCCGA-3', 5'-AGCTTCTCGGCTTTCAGGTC-3'; GPAT, 5'-TCCTAGCTCGCGATTTCGAC-3' ${ }^{\prime}$ 5'-ATCTTTCCTG CTCGTGTGGG-3'; Elovl6, 5'-TGCTGATGGGCTGTGTCATT-3' ${ }^{\prime}$ - 5'-GGAGTAGCACTGGTCGTTGT-3'; G0S2, 5'-AAAGTGTGCAGGAGCTGATC-3', 5'-GGACTGCTGTTCACACGCTT-3' and Rplp0, 5'-GCAGGTGTTT GACAACGGCA G-3', 5'-GATGATGGAGTGTGGCACCG A-3'.

Western blot analysis. For protein preparation from liver tissues, mouse livers (50 mg) were placed in a glass homogenizer containing $1 \mathrm{ml}$ Pro-Prep Protein Extraction Solution (Intron Biotechnology, Korea). Tissue lysates were separated by SDS-PAGE. Primary antibodies against SIRT1, PPAR $\gamma$ (Santa Cruz Biotechnology, Santa Cruz, CA), acetylated lysine (Cell Signaling Technology, Danvers, MA), and $\beta$-actin were used. Bands were detected with anti-rabbit or anti-mouse IgG conjugated with horseradish peroxidase (Pierce) using the ECL-PLUS detection system (Amersham, Little Chalfont, UK).

Primary hepatocyte culture. Primary mouse hepatocytes were isolated using the two-step collagenase perfusion method from the livers of male C57BL/6 (8 weeks old) mice as previously described ${ }^{35}$. Hepatocytes were plated onto six-well dishes at $1.0 \times 10^{6}$ cells per well and incubated for $12 \mathrm{~h}$ in DMEM containing $10 \%$ FBS to allow cells to attach. Cell counts and viability (Adam cell counter; Digital Bio) were confirmed before use. Viability was routinely $>85 \%$. After attachment, cells were infected with PPAR $\gamma 2$ - and SIRT1-expressing adenoviruses.

Preparation of recombinant adenovirus. Murine PPAR $\gamma 2$ and SIRT 1 cDNAs were cloned into the pcDNA3 or FLAG-tagged pcDNA3 vectors, respectively. Recombinant adenovirus (Ad) expressing PPAR $\gamma 2$ and ad-shRNA for MGAT1 were prepared as described ${ }^{15}$. All viruses were propagated in 293A cells and purified by $\mathrm{CsCl}$ density purification, dissolved in $1 \mathrm{x} \mathrm{HBSS}$ (Invitrogen), and stored at $-70^{\circ} \mathrm{C}$. The multiplicity of infection (MOI) was calculated from viral particle numbers. Recombinant adenovirus containing the GFP gene or Ad-US control RNAi were used as controls.

TG and cholesterol assay in the liver. Liver extracts (from $0.2 \mathrm{~g}$ tissues) were prepared by homogenization in chloroform:methanol $(2: 1, \mathrm{v} / \mathrm{v})$. TG and cholesterol levels were measured using TG assay or cholesterol assay reagents (ThermoFisher Scientific, Waltham, MA). The TG level was calculated from measurements of the absorbance at $500 \mathrm{nM}$ and expressed as $\mathrm{mg}$ TG/g liver wet weight.

Transfection and luciferase assay. HepG2 cells were transfected with the pGL3-MGATs promoter plasmid $^{15,20}$ as indicated or pRL-CMV (Promega, Madison, WI) using Lipofectamine (Invitrogen) according to the manufacturer's instructions. After $24 \mathrm{~h}$, luciferase activity was measured using the Dual Luciferase Reporter Assay System (Promega) according to the manufacturer's instructions. Firefly luciferase activities were standardized to Renilla activities.

$\mathrm{NAD}^{+} / \mathrm{NADH}$ assay. The $\mathrm{NAD}^{+} / \mathrm{NADH}$ ratio was measured using $\mathrm{NAD} / \mathrm{NADH}$ assay kits from Abcam according to the manufacturer's instructions. Absorbance was measured at $450 \mathrm{~nm}$ using the multi-detection reader (VERSA max, Molecular Devices, Sunnyvale, CA).

Immunoprecipitation and PPAR $\gamma$ acetylation. For immunoprecipitation, we lysed tissues using a passive lysis buffer (50 mM Tris- $\mathrm{HCl}, \mathrm{pH} 7.4,1 \% \mathrm{NP}-40,0.25 \%$ sodium deoxycholate, $150 \mathrm{mM} \mathrm{NaCl}, 1 \mathrm{mM}$ EDTA, and protease inhibitor cocktail). Proteins were immunoprecipitated with anti-PPAR $\gamma$ antibody (Santa Cruz Biotechnology), collected with protein A/G conjugated agarose beads (Santa Cruz Biotechnology), and washed three times with lysis buffer. Acetylation of immunoprecipitated proteins was assessed using anti-acetylated lysine antibody (Cell Signaling Technology). Western blots for PPAR $\gamma$ were also performed to assess the total protein quantity.

Immunocytochemistry. Primary mouse hepatocytes were isolated from the livers of wildtype or SIRT1 transgenic mice (10 weeks old, male) and then transfected with Ad-GFP or Ad-PPAR $\gamma 2$. At the indicated times, cells were washed with PBS, fixed in $4 \%$ formaldehyde for $15 \mathrm{~min}$, permeabilized with $0.2 \%$ Triton X-100 for 20 min on ice, and then blocked in 3\% bovine serum albumin in PBS for $1 \mathrm{~h}$. Cells were then incubated in a blocking solution containing ADRP antibody (1:200 dilution) for $12 \mathrm{~h}$, followed by fluorescein isothiocyanate-conjugated anti-mouse IgG secondary antibody for $2 \mathrm{~h}$. The cells were mounted in 4',6-diamidino-2-phenylindole. Cells were then visualized with a Confocal Laser Scanning Microscope (Olympus FV1000).

Statistical analysis. All data are expressed as the mean \pm SD with $n$ representing the number of analyzed mice. Statistical significance of observed differences between groups was determined using the unpaired Student's $t$-test. $\mathrm{P}<0.05$ was considered as statistically significant. 


\section{References}

1. Gao, B. \& Bataller, R. Alcoholic liver disease: pathogenesis and new therapeutic targets. Gastroenterology 141, 1572-1585 (2011).

2. Livero, F. A. \& Acco, A. Molecular basis of alcoholic fatty liver disease: From incidence to treatment. Hepatol. Res. 46, 111-123 (2015).

3. Marmier, S. et al. Novel role for carbohydrate responsive element binding protein in the control of ethanol metabolism and susceptibility to binge drinking. Hepatology 62, 1086-1100 (2015).

4. Rodgers, J. T. et al. Nutrient control of glucose homeostasis through a complex of PGC-1 alpha and SIRT1. Nature 434, 113-118 (2005).

5. Chalkiadaki, A. \& Guarente, L. Sirtuins mediate mammalian metabolic responses to nutrient availability. Nat. Rev. Endocrinol. 8, 287-296 (2012)

6. Houtkooper, R. H., Pirinen, E. \& Auwerx, J. Sirtuins as regulators of metabolism and healthspan. Nat. Rev. Mol. Cell. Biol. 13, 225-238 (2012)

7. Yin, H. Q. et al. Deletion of SIRT1 From Hepatocytes in Mice Disrupts Lipin-1 Signaling and Aggravates Alcoholic Fatty Liver. Gastroenterology 146, 801-811 (2014).

8. Picard, F. et al. Sirtl promotes fat mobilization in white adipocytes by repressing PPAR-gamma. Nature 429, 771-776 (2004).

9. Qiang, L. et al. Brown remodeling of white adipose tissue by SirT1-dependent deacetylation of Ppargamma. Cell 150, 620-632 (2012).

10. Semple, R. K., Chatterjee, V. K. K. \& O’Rahilly, S. PPAR gamma and human metabolic disease. J. Clin. Invest. 116, 581-589 (2006).

11. Lee, Y. J., Yu, J. H., Kim, W. H. \& Kim, J. W. New mechanisms contributing to hepatic steatosis: glucose, insulin, and lipid signaling. Anim. Cells. Syst. 18, 77-82 (2014).

12. Schadinger, S. E., Bucher, N. L., Schreiber, B. M. \& Farmer, S. R. PPARgamma2 regulates lipogenesis and lipid accumulation in steatotic hepatocytes. Am. J. Physiol. Endocrinol. Metab. 288, E1195-1205 (2005).

13. Vidal-Puig, A. et al. Regulation of PPAR gamma gene expression by nutrition and obesity in rodents. J. Clin. Invest. 97, 2553-2561 (1996).

14. Yamauchi, T. et al. Inhibition of RXR and PPARgamma ameliorates diet-induced obesity and type 2 diabetes. J. Clin. Invest. 108, 1001-1013 (2001).

15. Lee, Y. J. et al. Nuclear receptor PPARgamma-regulated monoacylglycerol O-acyltransferase 1 (MGAT1) expression is responsible for the lipid accumulation in diet-induced hepatic steatosis. Proc. Natl. Acad. Sci. USA. 109, 13656-13661 (2012).

16. Yen, C. L., Stone, S. J., Cases, S., Zhou, P. \& Farese, R. V. Jr. Identification of a gene encoding MGAT1, a monoacylglycerol acyltransferase. Proc. Natl. Acad. Sci. USA. 99, 8512-8517 (2002).

17. Hall, A. M. et al. Abrogating Monoacylglycerol Acyltransferase Activity in Liver Improves Glucose Tolerance and Hepatic Insulin Signaling in Obese Mice. Diabetes 63, 2284-2296 (2014).

18. Hayashi, Y. et al. Hepatic Monoacylglycerol O-acyltransferase 1 as a Promising Therapeutic Target for Steatosis, Obesity, and Type 2 Diabetes. Mol. Ther-Nucl. Acids 3 (2014).

19. Jaffe, H. A. et al. Adenovirus-mediated in vivo gene transfer and expression in normal rat liver. Nat. Genet. 1, 372-378 (1992).

20. Yu, J. H. et al. Monoacylglycerol O-acyltransferase 1 is regulated by peroxisome proliferator-activated receptor gamma in human hepatocytes and increases lipid accumulation. Biochem. Bioph. Res. Co. 460, 715-720 (2015).

21. Barrio, E. et al. Liver disease in heavy drinkers with and without alcohol withdrawal syndrome. Alcohol Clin. Exp. Res. 28, 131-136 (2004).

22. Semple, R. K., Chatterjee, V. K. \& O’Rahilly, S. PPAR gamma and human metabolic disease. J. Clin. Invest. 116, 581-589 (2006).

23. Rahimian, R. et al. Hepatic over-expression of peroxisome proliferator activated receptor gamma2 in the ob/ob mouse model of non-insulin dependent diabetes mellitus. Mol. Cell. Biochem. 224, 29-37 (2001).

24. Ahmadian, M. et al. PPARgamma signaling and metabolism: the good, the bad and the future. Nat. Med. 19, 557-566 (2013).

25. Hu, E. D., Kim, J. B., Sarraf, P. \& Spiegelman, B. M. Inhibition of adipogenesis through MAP kinase-mediated phosphorylation of PPAR gamma. Science 274, 2100-2103 (1996).

26. Compe, E. et al. Dysregulation of the peroxisome proliferator-activated receptor target genes by XPD mutations. Mol. Cell. Biol. 25, 6065-6076 (2005).

27. Choi, J. H. et al. Anti-diabetic drugs inhibit obesity-linked phosphorylation of PPARgamma by Cdk5. Nature 466, 451-456 (2010).

28. Shimizu, M., Yamashita, D., Yamaguchi, T., Hirose, F. \& Osumi, T. Aspects of the regulatory mechanisms of PPAR functions: analysis of a bidirectional response element and regulation by sumoylation. Mol. Cell. Biochem. 286, 33-42 (2006).

29. Hauser, S. et al. Degradation of the peroxisome proliferator-activated receptor gamma is linked to ligand-dependent activation. J. Biol. Chem. 275, 18527-18533 (2000).

30. Jiang, X., Ye, X., Guo, W., Lu, H. \& Gao, Z. Inhibition of HDAC3 promotes ligand-independent PPARgamma activation by protein acetylation. J. Mol. Endocrinol. 53, 191-200 (2014).

31. Ponugoti, B. et al. SIRT1 deacetylates and inhibits SREBP-1C activity in regulation of hepatic lipid metabolism. J. Biol. Chem. 285, 33959-33970 (2010).

32. Sugden, M. C., Caton, P. W. \& Holness, M. J. PPAR control: it’s SIRTainly as easy as PGC. J. Endocrinol. 204, 93-104 (2010).

33. Anstee, Q. M., Daly, A. K. \& Day, C. P. Genetics of Alcoholic and Nonalcoholic Fatty Liver Disease. Semin. Liver Dis. 31, 128-146 (2011).

34. Pessayre, D., Mansouri, A. \& Fromenty, B. Nonalcoholic steatosis and steatohepatitis v. mitochondrial dysfunction in steatohepatitis. Am. J. Physiol-Gastr. Liver Physiol. 282, G193-G199 (2002).

35. Kim, J. W. \& Ahn, Y. H. CCAAT/enhancer binding protein regulates the promoter activity of the rat GLUT2 glucose transporter gene in liver cells. Biochem. J. 336, 83-90 (1998).

\section{Acknowledgements}

This work was supported by the National Research Foundation of Korea (NRF) Grants 2011-0030086 and 2015R1A2A2A01004345, funded by the Korean government, Ministry of Science, ICT and Future Planning (MSIP).

\section{Author Contributions}

J.H.K., K.S.L. and J.-w.K. conceived and designed the experiments. J.H.Y., S.J.S., A.K., Y.C., J.W.S., H.J.K. and Y.J.L. performed the experiments. J.H.Y., H.J.K., and J.-w.K. analyzed data. J.H.Y., J.-w.K. wrote the paper.

\section{Additional Information}

Supplementary information accompanies this paper at http://www.nature.com/srep

Competing financial interests: The authors declare no competing financial interests.

How to cite this article: Yu, J. H. et al. Suppression of PPAR $\gamma$-mediated monoacylglycerol $O$-acyltransferase 1 expression ameliorates alcoholic hepatic steatosis. Sci. Rep. 6, 29352; doi: 10.1038/srep29352 (2016). 
(c) (i) This work is licensed under a Creative Commons Attribution 4.0 International License. The images or other third party material in this article are included in the article's Creative Commons license, unless indicated otherwise in the credit line; if the material is not included under the Creative Commons license, users will need to obtain permission from the license holder to reproduce the material. To view a copy of this license, visit http://creativecommons.org/licenses/by/4.0/ 